\title{
Modelling stellar activity cycles using deep-seated dynamos and surface flux transport
}

\author{
Emre Işık ${ }^{1}$, Dieter Schmitt ${ }^{2}$, and Manfred Schüssler ${ }^{2}$ \\ ${ }^{1}$ Department of Physics, Istanbul Kültür University, Bakırköy, 34156, Istanbul, Turkey \\ email: e.isik@iku.edu.tr \\ ${ }^{2}$ Max-Planck-Institut für Sonnensystemforschung, Max-Planck-Str. 2, 37191 \\ Katlenburg-Lindau, Germany
}

\begin{abstract}
We investigate the relations between tachocline-based dynamos and the surface flux transport mechanisms in stars with outer convection zones. Using our combined models of flux generation and transport, we demonstrate the importance of the buoyant rise of magnetic flux, which physically determines the emergence latitudes and tilt angles of bipolar magnetic regions. The combined effects of the dynamo strength, flux rise, and surface transport lead to various cyclic and non-cyclic time series of total unsigned surface magnetic flux.
\end{abstract}

Keywords. Sun: interior, stars: late-type, stars: magnetic fields

The outer layers of G- and K-type stars exhibit turbulent convection, which couples with rotation to amplify, transport, and disperse magnetic fields. A combination of various effects of magnetic flux transport can lead to a diversity of magnetic behaviour in $\mathrm{G}$ and $\mathrm{K}$ stars with different rotation rates. We have recently applied our three-part composite model (Işık et al. 2007) to investigate the effects of flux generation, rise, and transport in G-K main-sequence and evolved stars (Işık et al. 2011), which we briefly summarise below.

In the reference model corresponding to the Sun, we used a solar convection zone (CZ) model of Skaley \& Stix (1991) with an overshoot layer at the base. In this layer, we solved the dynamo equations numerically in $1 \mathrm{D}$ for both $\alpha$ and $\Omega$ effects operating co-spatially, with the nonlinear saturation level set by the buoyancy instabilities of toroidal flux tubes, which form in the same layer (Schmitt \& Schüssler 1989). The resulting butterfly diagram for the toroidal field determines the probability density for flux tube eruption. Flux tubes on the limit of buoyancy instability then rise through the $\mathrm{CZ}$, giving the emergence latitudes and tilt angles of bipolar regions at the surface. The emergent bipolar regions with solar area distribution are then transported by a surface flux transport model.

We found that with increasing rotation rate the dynamo cycle period shortens, and the correlation between the deep-CZ overshoot dynamo and the surface emergence pattern is gradually lost. For cooler stars, deeper convection zones decrease this correlation even further. Moreover, surface flux transport effects are also important in determining cycle properties. For a Sun-like star with a rotation period of 9 days, we found non-cyclic surface activity, in spite of the cyclic dynamo in the interior.

In the case of a K1-type subgiant star with the surface differential rotation, mass, and radius adopted from the K1IV component of HR 1099, we found that surface transport effects can lead to erratic variations in surface-integrated magnetic flux, which can be assumed to be correlated with stellar brightness (Figs. 1, 2). In this model, a multiperiodic cycle pattern with periodicities similar to that of HR 1099 has been found 

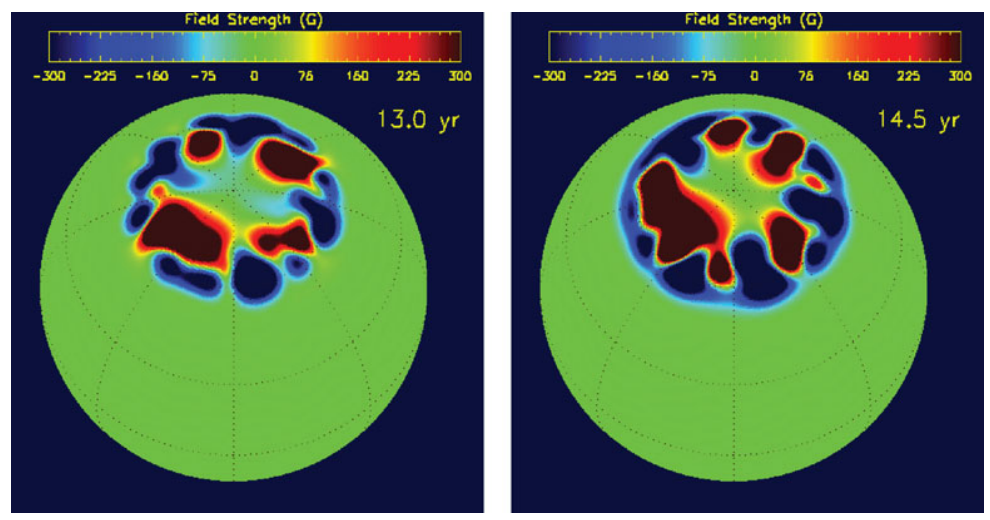

Figure 1. Magnetic field distribution in a K1-type subgiant star similar to the active component of HR 1099, during activity minimum (left) and maximum (right).

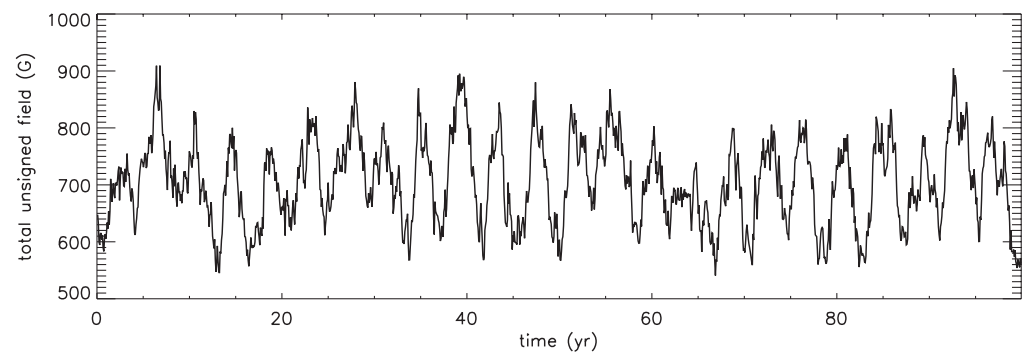

Figure 2. Variation of the total unsigned flux density for the K1-type subgiant star in Fig. 1.

(Oláh et al. 2009, Işı 2012). The mechanism responsible for the long-term variation in our model is that the stochastic emergence effects are of comparable importance with the surface transport effects and the dynamo-driven cyclic changes of polarity orientation of emerging bipolar regions.

In the light of our numerical simulations, we underline the importance of non-local and nonlinear effects such as buoyant rise of flux tubes, particularly for lower Rossby numbers. However, our models do not include the effect of the surface fields on the deep interior. To close the loop, our next attempt in stellar dynamo modelling is to construct a 2D Babcock-Leighton type dynamo model including rising flux tube simulations in stars with different rotation rates and convection zone depths.

\section{References}

Işık, E., Schmitt, D., \& Schüssler, M. 2007, AN, 328, 1111

Işık, E., Schmitt, D., \& Schüssler, M. 2011, A\&广A, 528, A135

Işık, E. 2012, in: C.H. Mandrini \& D. F. Webb (eds.), Comparative Magnetic Minima: Characterizing Quiet Times in the Sun and Stars, Proc. IAU Symposium No. 286 (Cambridge University Press), p. 291

Oláh, K., Kolláth, Z., Granzer, T., et al. 2009, A\&A, 501, 703

Schmitt, D. \& Schüssler, M. 1989, A\& A, 223, 343

Skaley, D. \& Stix, M. 1991, A\&A A, 241, 227 